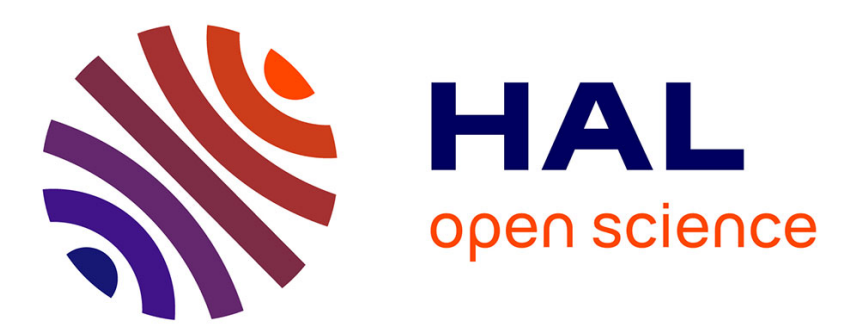

\title{
Dramatic decrease of Joule losses in the coupling between a crystalline gold film and single colloidal CdSe/CdS nanocrystals at $4 \mathrm{~K}$
}

\author{
A. Coste, L. Moreaud, G. Colas Des Francs, S. Buil, X. Quélin, Erik Dujardin, \\ J.-P. Hermier
}

\section{To cite this version:}

A. Coste, L. Moreaud, G. Colas Des Francs, S. Buil, X. Quélin, et al.. Dramatic decrease of Joule losses in the coupling between a crystalline gold film and single colloidal CdSe/CdS nanocrystals at 4 K. Physical Review B, 2020, 101 (7), 10.1103/PhysRevB.101.075406 . hal-02530759

\section{HAL Id: hal-02530759 \\ https://hal.science/hal-02530759}

Submitted on 1 Dec 2020

HAL is a multi-disciplinary open access archive for the deposit and dissemination of scientific research documents, whether they are published or not. The documents may come from teaching and research institutions in France or abroad, or from public or private research centers.
L'archive ouverte pluridisciplinaire HAL, est destinée au dépôt et à la diffusion de documents scientifiques de niveau recherche, publiés ou non, émanant des établissements d'enseignement et de recherche français ou étrangers, des laboratoires publics ou privés. 


\title{
Dramatic decrease of Joule losses in the coupling between a crystalline gold film and single colloidal $\mathrm{CdSe} / \mathrm{CdS}$ nanocrystals at $4 \mathrm{~K}$
}

\author{
A. Coste, ${ }^{1}$ L. Moreaud $\odot,{ }^{2}$ G. Colas des Francs $\odot,{ }^{3}$ S. Buil,${ }^{1}$ X. Quélin, ${ }^{1}$ E. Dujardin $\odot,{ }^{2}$ and J.-P. Hermier ${ }^{1, *}$ \\ ${ }^{1}$ Université Paris-Saclay, UVSQ, CNRS, GEMaC, 78000 Versailles, France \\ ${ }^{2}$ CEMES/CNRS UPR 8011, 29 rue Jeanne Marvig, 31055 Toulouse Cedex, France \\ ${ }^{3}$ Laboratoire Interdisciplinaire Carnot de Bourgogne (ICB), UMR 6303 CNRS, Université Bourgogne Franche-Comté, \\ 9 Avenue Savary, BP 47870, 21078 Dijon Cedex, France
}

(Received 18 July 2019; revised manuscript received 6 January 2020; accepted 8 January 2020; published 4 February 2020)

\begin{abstract}
We study the coupling of colloidal thick-shell CdSe/CdS nanocrystals with amorphous gold films (AFs) and crystalline gold platelets (CPs) at $4 \mathrm{~K}$. Time resolved experiments show that the photoluminescence lifetime increases from $2.4 \mathrm{~ns}(\mathrm{AF})$ to $5.1 \mathrm{~ns}(\mathrm{CP})$ due to the reduction of Joule losses. The comparison of the experimental results with numerical predictions shows that the radiative quantum efficiency is at least twice higher $(\geqslant 20 \%)$ for the crystalline structure.
\end{abstract}

DOI: 10.1103/PhysRevB.101.075406

\section{INTRODUCTION}

Since their first synthesis in 2008 [1,2], colloidal semiconductor $\mathrm{CdSe} / \mathrm{CdS}$ nanocrystals (NCs) with a thick shell have attracted a great interest. The major effect of the thick shell is to suppress the long off-fluorescence periods ubiquitous in single NC experiments [3].

Additionally, the thick shell acts as a highly efficient spacer when such NCs are coupled to plasmonic structures and minimizes the emission quenching by excitation of lossy surface plasmons and subsequent ohmic dissipation. In spite of this, the radiative quantum efficiency rarely exceeds a few percent at visible wavelengths [4].

Generally, the reduction of Joule losses in plasmonic structures can be obtained by lowering the temperature or minimizing the surface roughness. At $4 \mathrm{~K}$, electron-phonon scattering is strongly reduced and the propagation length of surface plasmons increases [5,6]. Recently, we have demonstrated the reduction of ohmic losses when thick-shell NCs are coupled to an evaporated gold film at $4 \mathrm{~K}$ [4]. Between 293 and $11 \mathrm{~K}$, the fluorescence decay rate is reduced by a factor of 2.3 while the radiative quantum efficiency is three times higher.

Alternatively, reducing the surface roughness or increasing the crystallinity of the plasmonic structure strongly reduces the damping of the surface plasmon polariton $[7,8]$.

In this paper, we combine cryogenic temperature operation and the use of single crystalline gold microplates to further reduce the Joule losses. Individual $\mathrm{CdSe} / \mathrm{CdS} \mathrm{NCs}$ are coupled to an amorphous film or a large single crystalline gold microplates of similar thickness and probed by time resolved photoluminescence at $4 \mathrm{~K}$. We show that the mean lifetime increases from $2.4 \mathrm{~ns}$ (amorphous film) to $5.1 \mathrm{~ns}$ (crystalline microplates). From these values and theoretical predictions,

*jean-pierre.hermier@uvsq.fr we deduce that the radiative quantum efficiency is at least twice higher in the case of a crystalline structure.

\section{EXPERIMENTS}

\section{A. Samples}

The amorphous flat gold films (AFs) were fabricated under ultrahigh vacuum $\left(10^{-9}\right.$ torr) by thermal evaporation of gold on a cleaned glass coverslip (1 in. diameter) at room temperature. The duration of the deposition is fixed so that the gold mass, measured with a quartz balance, corresponds to a thickness of $25 \mathrm{~nm}$. The roughness is determined by atomic force microscopy. For a $1 \mu \mathrm{m} \times 1 \mu \mathrm{m}$ surface, the RMS roughness is $1.1 \mathrm{~nm}$.

The crystalline gold microplates (CPs, Fig. 1) are synthesized by the chemical reduction of tetrachloroaurate(III) salts in the presence of aniline [9]. A 10-mM solution of $\mathrm{Au}(\mathrm{III})$ salts in ethylene glycol (EG, $18.2 \mathrm{~mL}$ ) is heated under stirring for $20 \mathrm{~min}$ prior to the addition of $180 \mu \mathrm{L}$ of $0.1 \mathrm{M}$ aniline. The solution is then stored unstirred for one day at room temperature. The resulting microplates have sizes in the range from $500 \mathrm{~nm}$ to approximately $8 \mu \mathrm{m}$. By centrifuging the suspension at $4300 \mathrm{~g}$ for $2 \mathrm{~min}$, the size distribution can be narrowed down to 1-5 $\mu \mathrm{m}$. The thickness of the microplates measured by atomic force microscopy (AFM) is $25 \pm 5 \mathrm{~nm}$. The excess of EG and aniline is removed by centrifugation and redispersion in a 10/90 ethanol/water mixture. The suspension is drop-casted onto a precleaned ITO-coated glass slide in order to obtain a uniform density of approximately one microplate per $10 \times 10 \mu \mathrm{m}^{2}$ area. The adsorbed EG and aniline capping layer are removed by a mild oxygen plasma treatment. The rms roughness of the plasma-cleaned microplates is $0.65 \pm 0.15 \mathrm{~nm}$.

The CdSe/CdS core-shell NCs are chemically synthesized following the protocol described in [10]. The core diameter is $3 \mathrm{~nm}$ while the shell thickness is $8 \mathrm{~nm}$. The ligands 


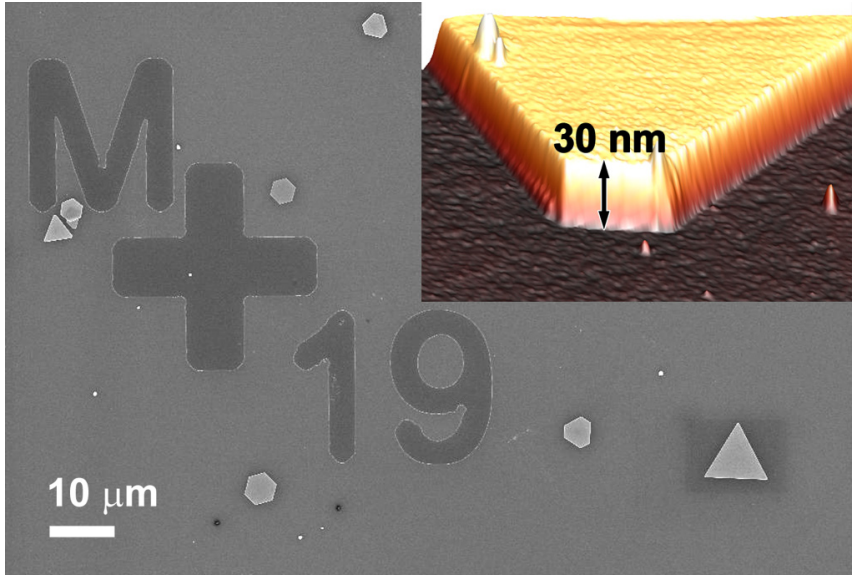

FIG. 1. SEM image of plasma-cleaned microplates on ITO/glass substrate. Inset: three-dimensional (3D) view of an AFM image of a 30-nm-thick microplate.

surrounding the individuals NCs have a mean length of $2 \mathrm{~nm}$. The shell and the ligands act as a spacer and we model in the following the single NCs as a degenerate dipole [11] localized $11 \mathrm{~nm}$ above the surface they were spin-casted on. The mean emission wavelength is respectively $620 \mathrm{~nm}$ at room temperature and $590 \mathrm{~nm}$ at $4 \mathrm{~K}$.

\section{B. Experimental setups}

The NC stock solution is diluted and spin coated on a glass coverslip, the $\mathrm{AF}$ or the $\mathrm{CP}$ so that single NCs fluorescence can be characterized with a confocal microscope. The NCs were deposited using the same protocol. Since the bare gold surface of $\mathrm{CP}$ is plasma cleaned while the evaporated AFs are obtained under ultravacuum, we can assume that the distance $d$ between the NCs and the CP surface and the AF surface are identical. A cryogen-free setup (Cryostat attoDRY1100, low-temperature confocal microscope attoCFM-I, Attocube, numerical aperture objective $=0.82$ ) provides measurements without any drift for several hours. Optical excitation is obtained with a pulsed supercontinuum laser (NKT Photonics SuperK EXTREME, $\lambda=485 \mathrm{~nm}$, pulse duration $\sim 150 \mathrm{ps}$ ). The emission is detected with an avalanche photodiode (MPD, $50 \mathrm{ps}$ time resolution) linked to a counting module (PicoHarp 300 module, Picoquant, 64 ps time resolution). The instrumental function response (IRF) of the experimental setup is then of the order of $200 \mathrm{ps}$.

\section{RESULTS AND DISCUSSION}

In all the following experiments, low power excitation (mean power $\sim 400 \mathrm{nW}$, pulse width $\sim 200 \mathrm{ps,} \mathrm{repetition} \mathrm{rate}$ $=20 \mathrm{MHz}$ ) is used such that the probability to generate one electron-hole pair per pulse prevails. The photoluminescence (PL) decay of single NCs deposited on a glass coverslip is first characterized at $4 \mathrm{~K}$ [Fig. 2(a)]. Under air and at room temperature, $\mathrm{CdSe} / \mathrm{CdS} \mathrm{NCs}$ are known to switch between a neutral and an ionized state. However, under vacuum, thickshell NCs deposited on a glass coverslip remain ionized [12]. This observation is first explained by the removal of ligands induced by the vacuum generation and the resulting apparition

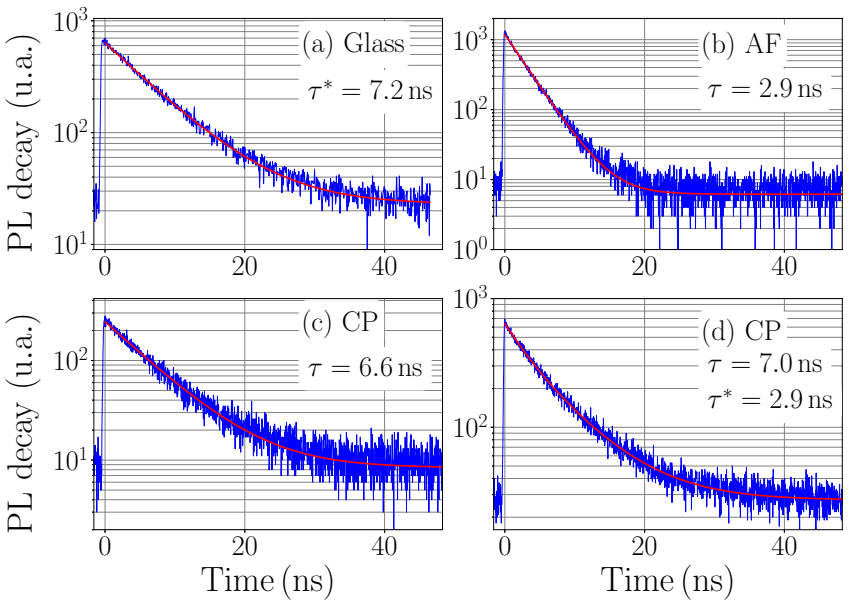

FIG. 2. PL decays of single NCs on glass (a), AF (b), and CP (c),(d). The NC remains in the ionized state (a) or in the neutral state (b),(c). The red lines represent monoexponential fits providing the trion lifetime (a) and exciton lifetime (b),(c). For (d), the NC switches between the neutral and the ionized states and biexponential decay is measured (red line)

of dangling bonds that cannot be saturated by the atmosphere. Moreover, ionized states become very stable since no neutralization can occur through charge transfer between air molecules and the surface of the NC $[13,14]$. At $4 \mathrm{~K}$, the Auger recombination of the trion is also inhibited which leads to a pure radiative recombination. We then find that the mean radiative lifetime of the trion on a glass coverslip is $6.8 \pm 1.2 \mathrm{~ns}$ (average over $18 \mathrm{NCs}$ ).

We now compare this result to the ones obtained when the NCs are coupled to the AF or the CP. In contrast, $\mathrm{NCs}$ coupled to the AF or the $\mathrm{CP}$ are not systematically ionized. This can be explained by taking into account the work function (WF) of gold as well as the $\mathrm{CdS}$ and $\mathrm{SiO}_{2}$ electron affinities (EAs) and ionization energies (IEs) [15]. For gold, the WF ranges between 4.6 and $5.5 \mathrm{eV}$ depending on parameters such as crystallinity, roughness, and exposition to air $[15,16]$. These values are close to the EA and IE of bulk CdS which are respectively 4.7 and $7.1 \mathrm{eV} \mathrm{[17]} \mathrm{as} \mathrm{confirmed}$ by the measurement of a small barrier height $(\sim 0.5 \mathrm{eV})$ of $\mathrm{Au} / \mathrm{CdS} / \mathrm{SnO}_{2} / \mathrm{In}-\mathrm{Ga}$ structures [18]. Even if this analysis does not take into account the nanometric size of the CdS shell and the presence of ligands, these data suggest that charge exchanges between the NCs and the surface are eased in the case of a gold film. On the contrary, the IE of $\mathrm{SiO}_{2}$, that is an insulator, is as high as $8 \mathrm{eV}$ while the EA is as low as $0.9 \mathrm{eV}$ [19], meaning that $\mathrm{SiO}_{2}$ cannot provide a charge (electron or hole) to an ionized $\mathrm{NC}$ with a $\mathrm{CdS}$ shell. Under vacuum, neutralization after photoionization of the NC can then occur on the gold structures in strong contrast with the silica coverslip. Experimentally, we observed that the neutralization process is more efficient in the case of AFs when compared to CPs. Moreover, a nearby plasmonic gold structure enhances the fluorescence processes compared to charge trapping outside the NC core [20]. The stability of the neutral state therefore increases as the exciton recombination occurs at a faster rate than the charge escape. 
The advantage of considering the excitonic state versus the trion one is that its decay rate is lower and its measurement is less affected by the time resolution of the setup. As already stated by Schwartz et al., the stability of the neutral state is all the greater as the repetition rate of the laser is lower [21]. In our experiments, we promote the neutral state stability by reducing the repetition rate of the laser to $20 \mathrm{MHz}$, for which we observe that the contribution of the exciton recombination becomes significant while a good signal-to-noise ratio is preserved.

In the following, the PL decay of single NCs is fitted by a single exponential when it remains neutral [Figs. 2(b) and 2(c)] or by a biexponential when it switches between neutral and ionized states [Fig. 2(d)]. For the AF, the mean excitonic lifetime is about $2.4 \pm 0.3 \mathrm{~ns}$ (average over $11 \mathrm{NCs}$ ). Since the decay rate of the trion is twice greater than the excitonic one in the absence of Auger recombination [22], this corresponds to a Purcell factor (defined as the decay rate enhancement due to the gold structure, taking the glass coverslip as the reference) $F_{p}$ of $2 \times 6.8 / 2.4=5.7 \pm 1.2$, a value close to the one we have previously reported for the decay rate enhancement for NCs of similar sizes deposited on a flat gold film at $4 \mathrm{~K}$ [4]. Importantly, the Purcell factors for the amorphous and crystalline structures show low dispersion that can be attributed to small dispersion of the distance between the NCs and the gold film $(11 \pm 1 \mathrm{~nm})$ originating from the size dispersion of NCs. This low dispersion ensures that the gap between the $F_{p}$ factors comes from the gold crystallinity.

When CPs are used, the position of the NCs is random. We considered the emitters located at a distance $d$ exceeding $d_{\text {min }}=1 \mu \mathrm{m}$ from the edges of the CPs. Otherwise, the surface plasmon polariton (SPP) launched by the NC could be reflected so that the CPs could not be compared to an infinite flat film. In the following, we will check this hypothesis considering the SPP propagation length $d_{\min }$ is high enough. The shape of the Au particle has then no effect.

The dramatic effect of the gold crystallinity on the coupling between a gold film and a single NC is first evidenced by the large increase of the mean exciton lifetime, which reaches $5.1 \pm 1.3 \mathrm{~ns}$ (average over $10 \mathrm{NCs})\left(F_{p}=2 \times 6.8 / 5.1=\right.$ $2.7 \pm 0.8$ ), suggesting a drastic reduction of the Joule losses for the CP.

Since the roughness of the AF and CP films is of the order of $1 \mathrm{~nm}$, its effect on the NCs fluorescence enhancement can be neglected. The coupling between the single NCs and the gold structures is modelled through the gold film dielectric constant $\epsilon$ alone. For gold $\epsilon$, values have been reported in several references $[23,24]$. However, the thickness as well as the elaboration process have to be considered [25]. $\epsilon$ also strongly depends on the temperature operation [7,26].

In the presence of the plasmonic film, one can distinguish three main relaxation channels for the dipolar emission: (i) radiative emission, (ii) surface plasmon coupled emission (SPCE) with the excitation of the Au/air and the Au/glass SPPs, and (iii) lossy waves. Considering a distribution of NCs showing a random orientation and using the Chance, Prock, and Silbey model $[27,28]$, we calculated the total decay rate $\Gamma_{\text {tot }}$, the radiative decay rate $\Gamma_{\text {rad }}$, and the SPCE rates $\Gamma_{\mathrm{SPP}}^{\mathrm{Au} / \mathrm{air}}, \Gamma_{\mathrm{SPP}}^{\mathrm{Au} / \mathrm{glass}}$ for a large range of values of the real $\epsilon_{r}$, and imaginary parts $\epsilon_{i}$ of the dielectric function $\epsilon$. Practically,

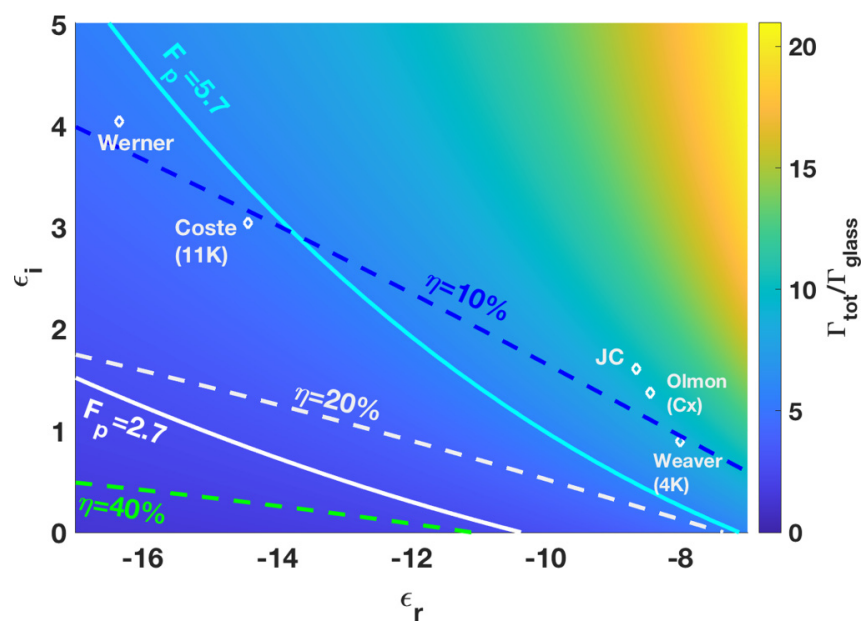

FIG. 3. Calculated Purcell factor $F_{p}=\Gamma_{\text {tot }} / \Gamma_{\text {glass }}$ as a function of the real and imaginary parts of the gold film dielectric function. Solid and dashed lines refer to iso- $F_{p}$ and iso-QE, respectively. Diamonds indicate some values of the gold dielectric function taken from the literature: Coste et al. [4], Johnson and Christy (JC) [23], Olmon et al. [29], Werner et al. [30], and Weaver et al. [31].

the total decay rate is numerically computed from the Weyl momentum spectrum representation of the dipolar emission that is an expansion over plane and evanescent waves. The radiative contribution is obtained by limiting the expansion to the plane waves only. Similarly, limiting the expansion to the SPP momentum, we estimate the SPCE rates. The corresponding Purcell factor is shown in Fig. 3.

In a previous study [4], we measured by spectrophotometry the real $\epsilon_{r}$ and imaginary parts $\epsilon_{i}$ of $\epsilon$ at $590 \mathrm{~nm}$ for AF (11 K) and found $\epsilon_{r}=-14.4$ and $\epsilon_{i}=3.0$ which gives $F_{P}=5.3$, in agreement with the experimental value of $F_{P}=5.7$. We also compute the far-field dipolar emission $\Gamma_{\text {scatt }}$ so that the radiative quantum efficiency $\left(\mathrm{QE}=\Gamma_{\text {scatt }} / \Gamma_{\text {tot }}\right)$ has been inferred to be equal to $10 \%$. A structuration of a crystalline gold surface could then be used to decouple the bound Au/glass SPP to the far field and increase the collection efficiency [32,33].

Concerning the CPs, they are too small to perform such spectrophotometric experiments. Only $F_{P}$ can be measured. However, the model enables us to plot the iso-QE and iso$F_{P}$ lines. Figure 3 shows that for $F_{P}=2.7$, the $\mathrm{QE}$ ranges between $20 \%$ and $40 \%$ for the considered range of $\epsilon_{r}$ and $\epsilon_{i}$. The $\mathrm{QE}$ for the $\mathrm{CP}$ is then at least twice larger when compared to the AF, confirming the drastic reduction of Joule losses. The estimated coupling rate to the gold/air SPP $\beta=$ $\Gamma_{\mathrm{SPP}}^{\mathrm{Au} / \mathrm{Air}} / \Gamma_{\text {tot }}$ also increases from about $8 \%$ for $\mathrm{AF}$ to $17 \%$ for CP. Finally, we also calculate that the propagation length gold/air SPP is around $2 \mu \mathrm{m}$. An upper value of the reflection coefficient of the SPP at the CP edge can be estimated as follows. First, a dielectric model for guided SPP [34] leads to a reflection coefficient at normal incidence of $R=\left(n_{\mathrm{spp}}-\right.$ $1) /\left(n_{\text {spp }}+1\right) \sim 2 \%$ where $n_{\text {spp }} \sim 1.03$ is the SPP effective index. In addition, an overestimated value of $20 \%$ can be estimated from plasmonic Bragg mirror [35]. Considering an order of magnitude of $20 \%$ for the reflection coefficient of the SPP at the CP edge, this $L_{\mathrm{SPP}}$ value implies that the relative amplitude of the reflected SPP is lower than $5 \%$ for a NC 
located at a distance $d_{\text {min }}$ greater than $1 \mu \mathrm{m}$ from the CP edge. This means that the CP can be considered as a flat film in our experiments.

\section{CONCLUSION}

In conclusion, our results demonstrate that a large increase of the QE of thick-shell $\mathrm{CdSe} / \mathrm{CdS}$ NCs coupled to ultrathin $2 \mathrm{D}$ gold structures is obtained at $4 \mathrm{~K}$ by using single crystalline gold microplates. While cryogenic temperature operation alone enables us to reach QE values close 10\%, the presented results demonstrate that the replacement of amorphous gold by crystalline gold enables us to exceed $20 \%$.

These results are particularly important and promising for further coupling NCs to plasmonic structures when quantum plasmonics applications are foreseen. Future efforts can now be devoted to fabricate structures that couple the surface plasmon emission into the light cone.

\section{ACKNOWLEDGMENTS}

E.D. and L.M. acknowledge the financial support of the Agence Nationale de la Recherche (Contract No. ANR16-CE09-0027-HybNaP). This work was also supported by the French "Investissements d'Avenir" program ISITE-BFC IQUINS (ANR-15-IDEX-03) and EUR-EIPHI (17-EURE0002). Calculations were performed using HPC resources from DSI-CCuB (Université de Bourgogne). The authors thank F. Dell' Ova for technical assistance in scanning electron microscope and AFM imaging of the microplates.
[1] B. Mahler, P. Spinicelli, S. Buil, X. Quélin, J.-P. Hermier, and B. Dubertret,Towards non-blinking colloidal quantum dots, Nat. Mater. 7, 659 (2008).

[2] Y. F. Chen, J. Vela, H. Htoon, J. L. Casson, D. J. Werder, D. A. Bussian, V. I. Klimov, and J. A. Hollingsworth, Y. Chen, "Giant" Multishell CdSe nanocrystal quantum dots with suppressed blinking, J. Am. Chem. Soc. 130, 5026 (2008).

[3] M. Kuno, D. P. Fromm, H. F. Hamann, A. Gallagher, and D. J. Nesbitt, Nonexponential 'blinking' kinetics of single CdSe quantum dots: A universal power law behavior, J. Chem. Phys. 112, 3117 (2000).

[4] A. Coste, F. Eloi, C. Arnold, G. Colas des Francs, X. Quélin, S. Buil, A. Bouhelier, J. C. Weeber, M. Nasilowski, B. Dubertret, and J.-P. Hermier, Significant decrease of the optical losses in the coupling between colloidal $\mathrm{CdSe} / \mathrm{CdS}$ nanocrystals and a flat gold film at cryogenic temperature, Phys. Rev. B 96, 195416 (2017).

[5] M. Mayy, G. Zhu, E. Mayy, A. Webb, and M. A. Noginov, Low temperature studies of surface plasmon polaritons in silver films, J. Appl. Phys. 111, 094103 (2012).

[6] S. V. Jayanti, J. H. Park, A. Dejneka, D. Chvostova, K. M. McPeak, X. Chen, S.-H. Oh, and D. J. Norris, Low-temperature enhancement of plasmonic performance in silver films, Opt. Mat. Express 5, 1147 (2015).

[7] M. Liu, M. Pelton, and P. Guyot-Sionnest, Reduced damping of surface plasmons at low temperatures, Phys. Rev. B 79, 035418 (2009)

[8] M. Bosman, L. Zhang, H. Duan, S. F. Tan, C. A. Nijhuis, C.-W. Qiu, and J. K. W. Yang, Encapsulated annealing: Enhancing the plasmon quality factor in lithographically-defined nanostructures, Sci. Rep. 4, 5537 (2014).

[9] Z. Guo, Y. Zhang, Y. Duan Mu, L. Xu, S. Xie, and N. Gua, Facile synthesis of micrometer-sized gold nanoplates through an aniline-assisted route in ethylene glycol solution, Colloids Surf., A 278, 33 (2006).

[10] B. Ji, E. Giovanelli, B. Habert, P. Spinicelli, M. Nasilowski, X. Xu, N. Lequeux, J.-P. Hugonin, F. Marquier, J.-J. Greffet, and B. Dubertret, Non-blinking quantum dot with a plasmonic nanoshell resonator, Nat. Nano. 10, 170 (2015).

[11] C. Lethiec, J. Laverdant, H. Vallon, C. Javaux, B. Dubertret, J.-M. Frigerio, C. Schwob, L. Coolen, and A. Maître, Measurement of Three-Dimensional Dipole Orientation of a Single
Fluorescent Nanoemitter by Emission Polarization Analysis, Phys. Rev. X 4, 021037 (2014).

[12] C. Javaux, B. Mahler, B. Dubertret, A. Shabaev, A. V. Rodina, Al. L. Efros, D. R. Yakovlev, F. Liu, M. Bayer, G. Camps, L. Biadala, S. Buil, X. Quélin, and J.-P. Hermier, Thermal activation of non-radiative Auger recombination in charged colloidal nanocrystals, Nat. Nanotechnol. 8, 206 (2013).

[13] D. E. Gómez, J. van Embden, P. Mulvaney, M. J. Fernée, and H. Rubinsztein-Dunlop, Exciton-trion transitions in single CdSeCdS core-shell nanocrystals, ACS Nano 3, 2281 (2009).

[14] J. Müller, J. M. Luptona, A. L. Rogach, and J. Feldmann, Air-induced fluorescence bursts from single semiconductor nanocrystals, Appl. Phys. Lett. 85, 381 (2004).

[15] A. Kahn, Fermi level, work function and vacuum level, Mat. Horizons 3, 7 (2016).

[16] G. N. Derry, M. E. Kern, and E. H. Worth, Recommended values of clean metal surface work function, J. Vac. Sci. Technol. A 33, 060801 (2015).

[17] V. Stevanović, S. Lany, D. S. Ginley, W. Tumas, and A. Zunger, Assessing capability of semiconductors to split water using ionization potentials and electron affinities only, Phys. Chem. Chem. Phys. 16, 3706 (2014).

[18] I. Taşçioğlu, Ş. Atlindal, I. Polat, and E. Bacaksiz, The effect of metal work function on the barrier height ofmetal/CdS/ SnO2/IneGa structures, Curr. Appl. Phys. 13, 1306 (2013).

[19] R. Williams, Photoemission of electrons form silicon into silicon dioxide, Phys. Rev. 140, A569 (1965).

[20] F. T. Rabouw, M. Kamp, R. J. A. van Dijk-Moes, D. R. Gamelin, A. F. Koenderink, A. Mejerink, and D. Vanmaekelbergh, Delayed exciton, emission and its relation to blinking in CdSe quantum dots, Nano Lett. 15, 7718 (2015).

[21] O. Schwartz, R. Tenne, J. M. Levitt, Z. Deutsch, S. Itzhakov, and D. Oron, Colloidal quantum dots as saturable fluorophores, ACS Nano 6, 8778 (2012).

[22] C. Galland, Y. Ghosh, A. Steinbrück, J. A. Hollingsworth, H. Htoon, and V. I. Klimov, Lifetime blinking in nonblinking nanocrystal quantum dots, Nat. Commun. 3, 908 (2012).

[23] P. B. Johnson and R. W. Christy, Optical constants of the noble metals, Phys. Rev. B 6, 4370 (1972).

[24] K. M. McPeak, S. V. Jayanti, S. J. Kress, S. Meyer, S. Iotti, A. Rossinelli, and D. J. Norris, Plasmonic films can easily be better: Rules and recipes, ACS Photon. 2, 326 (2015). 
[25] D. I. Yakubovsky, A. V. Arsenin, Y. V. Stebunov, D. Y. Fedyanin, and V. S. Volkov, Optical constants and structural properties of thin gold films, Opt. Express 25, 25574 (2017).

[26] S. K. Ozdemir, Temperature effects on surface plasmon resonance: Design considerations for an optical temperature sensor, J. Lightwave Technol. 21, 805 (2003).

[27] R. R. Chance, A. Prock, and R. Silbey, Molecular fluorescence and energy transfer near interfaces, Adv. Chem. Phys. 37, 1 (1978).

[28] G. Colas des Francs, J. Barthes, A. Bouhelier, J. C. Weeber, A. Dereux, A. Cuche, and C. Girard, Plasmonic purcell factor and coupling efficiency to surface plasmons. Implications for addressing and controlling optical nanosources, J. Opt. 18, 094005 (2016).

[29] R. L. Olmon, B. Slovick, T. W. Johnson, D. Shelton, S.-H. Oh, G. D. Boreman, and M. B. Raschke, Optical dielectric function of gold, Phys. Rev. B 86, 235147 (2012).

[30] W. Werner, K. Glantsching, and C. Ambrosch-Draxl, Optical constants and inelastic electron-scattering data for 17 elemental metals, J. Phys. Chem. Ref. Data 38, 1013 (2009).
[31] J. H. Weaver, C. Krafka, D. W. Lynch, and E. E. Koch, Physics Data: Optical Properties of Metals, Pt. II (Fachinformationszentrum Energie, Physik, Mathematik GmbH, Karlsruhe, Germany, 1981), pp. 65-81.

[32] U. Kumar, S. Viarbitskaya, A. Cuche, C. Girard, S. Bolisetty, R. Mezzenga, G. Colas des Francs, A. Bouhelier, and E. Dujardin, Designing plasmonic eigenstates for optical signal transmission in planar channel devices, ACS Photon. 5, 2328 (2018).

[33] J. S. Huang, V. Callegari, P. Geisler, C. Bruning, J. Kern, J. C. Prangsma, X. F. Wu, T. Feichtner, J. Ziegler, P. Weinmann, M. Kamp, A. Forchel, P. Biagioni, U. Sennhauser, and B. Hecht, Atomically flat single-crystalline gold nanostructures for plasmonic nanocircuitry, Nat. Commun. 1, 150 (2010).

[34] R. Zia, A. Chandran, and M. L. Brongersma, Dielectric waveguide model for guided surface polaritons, Opt. Lett. 30, 1473 (2005).

[35] S. Derom, A. Bouhelier, A. Kumar, A. Leray, J.-C. Weeber, S. Buil, X. Quelin, J. P. Hermier, and G. Colas des Francs, Single-molecule controlled emission in planar plasmonic cavities, Phys. Rev. B 89, 035401 (2014). 